\title{
THE MINIMAL OPERATOR MODULE OF A BANACH MODULE
}

\author{
by BOJAN MAGAJNA
}

(Received 23rd June, 1997)

\begin{abstract}
Banach modules over $C^{*}$-algebras (von Neumann algebras) that can be represented isometrically as operator modules (normal operator modules, respectively) are characterised.
\end{abstract}

1991 Mathematics subject classification: 46L05, 46L10.

\section{Introduction}

It is well known that each Banach space $X$ can be linearly isometrically embedded into a commutative $C^{*}$-algebra, hence $X$ can be regarded as an operator space. If $X$ has some additional algebraic structure, we may ask whether an embedding can be found which preserves this additional structure. For example, if $X$ is a Banach algebra, the criterion for $X$ to have a bicontinuous isomorphic representation as an algebra of operators on a Hilbert space was obtained in [20] (see also [3] and [5]). Here we shall consider the case when $X$ is a Banach bimodule over a pair of $C^{*}$-algebras $A$ and $B$. Using the abstract characterisation of operator bimodules developed by Christensen, Effros and Sinclair in [6] we show first that a normed $A, B$-bimodule can be represented isometrically as an operator bimodule if and only if

$$
\|a x b+c y d\| \leq\left\|a a^{*}+c c^{*}\right\|^{1 / 2} \max \{\|x\|,\|y\|\}\left\|b^{*} b+d^{*} d\right\|^{1 / 2}
$$

for all $a, c \in A, x, y \in X$ and $b, d \in B$. Although the characterisation in [6] can be extended to the case when $A$ and $B$ are general operator algebras (not necessarily $C^{*}$ algebras), we will show by a finite dimensional example that the above isometric characterisation cannot be extended to modules over general operator algebras.

If $A$ and $B$ are von Neumann algebras and $X$ is a Banach $A, B$-bimodule, we may ask under what conditions can $X$ be represented isometrically as a normal operator $A, B$-bimodule. More precisely, we would like to know when does there exist a Hilbert space $\mathcal{H}$, an isometry $\Phi: X \rightarrow \mathbf{B}(\mathcal{H})$ and a pair of normal representations $\pi: A \rightarrow \mathrm{B}(\mathcal{H})$ and $\sigma: B \rightarrow \mathrm{B}(\mathcal{H})$ such that $\Phi(a x b)=\pi(a) \Phi(x) \sigma(b)$ for all $a \in A, x \in X$ and $b \in B$. In this case we shall simply say that $X$ can be represented as a normal operator $A, B$-bimodule. In the case of one-sided modules (say if $B=\mathbb{C}$ ), we shall show that a Banach $A$-module $X$ satisfying the condition (1.1) can be represented as a normal operator $A$-module if and only if for each $x \in X$ the mapping $x \mapsto\|a x\|$ is 
lower semicontinuous on (the unit ball of) $A$ in the weak* topology. We obtain a similar condition for bimodules, but the role of $A$ is played by the Haagerup tensor product $A \otimes^{h} B$ and the weak* topology is replaced by the topology which is induced on $A \otimes^{h} B$ by all completely bounded bilinear forms on $A \times B$ which are normal in each variable separately.

Given a von Neumann algebra $A \subseteq X=\mathrm{B}(\mathcal{H})$ and a norm closed $A$-submodule $Y$ in $X$ the quotient module $X / Y$ is not necessarily representable as a normal operator module. A necessary condition for this is that $Y$ is closed in the so called $A$-topology of $X$ which was introduced in [12]. (We shall recall the definition of this topology in Section 4.) But in general the condition that $Y$ is a closed submodule of $X$ in the $A$ topology does not suffice for $X / Y$ to be representable as a normal operator module. However, if $X \supseteq A$ is a von Neumann algebra and $Y$ is the closure in the $A$-topology of an ideal in $X$, then we will show (as an application of the result mentioned in the previous paragraph) that $X / Y$ can be represented as a normal operator $A$-module.

Throughout the paper we denote by $\mathcal{M}_{m, n}(X)$ the set of all $m \times n$ matrices with the entries in a set $X(m, n \in \mathbb{N})$ and we let $\mathcal{M}_{n}(X)=\mathcal{M}_{n, n}(X), \mathcal{R}_{n}(X)=\mathcal{M}_{1, n}(X)$ and $\mathcal{C}_{n}(X)=\mathcal{M}_{n, 1}(X)$ be the corresponding sets of square matrices, rows and columns.

\section{A characterisation of operator modules among Banach modules}

If $A$ and $B$ are operator algebras with contractive approximate identities $\left\{e_{\mu}\right\}$ and $\left\{f_{v}\right\}$, respectively, then by a Banach $A, B$-bimodule we mean a Banach space $X$ which is an $A, B$-bimodule satisfying $\|a x b\| \leq\|a\|\|x\|\|b\|$ for all $a \in A, b \in B$ and $x \in X$; moreover we shall always assume that $\lim e_{\mu} x=x=x \lim f_{v}$ for all $x \in X$. Thus all the modules here are essential, which by the Cohen Hewitt factorization theorem means that $A X=X=X B$. If for each $n \in \mathbb{N}$ the space $\mathcal{M}_{n}(X)$ is equipped with a norm \|\| such that the norm on $\mathcal{M}_{1}(X)=X$ coincides with the given norm on $X$ and

$$
\|a x b\| \leq\|a\|\|x\|\|b\|
$$

for all $a, b \in \mathcal{M}_{n}(\mathbb{C}), x \in \mathcal{M}_{n}(X)$ and

$$
\left\|\left[\begin{array}{ll}
x & 0 \\
0 & y
\end{array}\right]\right\|=\max \{\|x\|,\|y\|\}
$$

for all $x \in \mathcal{M}_{m}(X)$ and $y \in \mathcal{M}_{n}(X)$ (all $m, n \in \mathbb{N}$ ), then $X$ can be regarded as an operator space by the Ruan theorem [17, 9]. Moreover, if the condition (i) holds for all $a \in \mathcal{M}_{n}(A), b \in \mathcal{M}_{n}(B)$ and $x \in \mathcal{M}_{n}(X)$ then we say that the bimodule multiplication $A \times X \times B \rightarrow X$ is completely contractive. If $A$ and $B$ are $C^{*}$-algebras and the bimodule multiplication is completely contractive then $X$ is an operator bimodule by a result of Christensen, Effros and Sinclair [6]. This means that there exist a Hilbert space $\mathcal{H}$, a complete isometry $\Phi: X \rightarrow \mathrm{B}(\mathcal{H})$ and two faithful *-representations $\pi: A \rightarrow \mathrm{B}(\mathcal{H})$ and $\sigma: B \rightarrow \mathrm{B}(\mathcal{H})$ such that $\Phi(a x b)=\pi(a) \Phi(x) \sigma(b)$ for all $a \in A, b \in B$ and $x \in X$. (For basic notions concerning completely bounded mappings we refer to [13] or [16].) 
Theorem 2.1. Let $A, B$ be $C^{*}$-algebras and $X$ a Banach $A, B$-bimodule. Then $X$ can be represented isometrically as an operator $A, B$-bimodule if and only if

$$
\left\|a_{1} x_{1} b_{1}+a_{2} x_{2} b_{2}\right\| \leq\left\|a_{1} a_{1}^{*}+a_{2} a_{2}^{*}\right\|^{1 / 2} \max \left\{\left\|x_{1}\right\|,\left\|x_{2}\right\|\right\}\left\|b_{1}^{*} b_{1}+b_{2}^{*} b_{2}\right\|^{1 / 2}
$$

for all $a_{i} \in A, b_{i} \in B, x_{i} \in X(i=1,2)$. If this condition is satisfied then the minimal operator $A, B$-bimodule norms on $\mathcal{M}_{n}(X)(n=1,2, \ldots)$ are given by

$$
\|x\|=\sup \left\{\|a x b\|: a \in \mathcal{R}_{n}(A), b \in \mathcal{C}_{n}(B),\|a\| \leq 1,\|b\| \leq 1\right\} .
$$

Proof. If $X$ is an operator $A, B$-bimodule, then in particular the module multiplication $\mathcal{R}_{2}(A) \times \mathcal{M}_{2}(X) \times \mathcal{C}_{2}(B) \rightarrow X$ is contractive, which implies the condition (2.1).

Conversely, if the condition (2.1) holds, then for each $n$ define a norm on $\mathcal{M}_{n}(X)$ by (2.2). It is easy to verify that (2.2) indeed defines a norm on $\mathcal{M}_{n}(X)$. The fact that $\|x\|=0$ implies $x=0$ follows since $A X B \neq 0$ (recall that we have assumed that all the modules here are essential). Moreover, it follows directly from (2.2) that the multiplication $\mathcal{M}_{m, n}(A) \times \mathcal{M}_{n}(X) \times \mathcal{M}_{n, m}(B) \rightarrow \mathcal{M}_{m}(X)$ is contractive and consequently (since $X$ is essential $A, B$-bimodule) the multiplication $\mathcal{M}_{m, n}(\mathbb{C}) \times \mathcal{M}_{n}(X) \times \mathcal{M}_{n, m}(\mathbb{C}) \rightarrow \mathcal{M}_{m}(X)$ is also contractive for all $m, n \in \mathbb{N}$. This implies in particular that

$$
\max \left\{\left\|x_{1}\right\|,\left\|x_{2}\right\|\right\} \leq\left\|x_{1} \oplus x_{2}\right\|
$$

for all $x_{i} \in \mathcal{M}_{n_{i}}(X)$, where $n_{i} \in \mathbb{N}, i=1,2$. (To see this, note for example, that $x_{1}=[I, 0]\left(x_{1} \oplus x_{2}\right)[I, 0]^{T}$, where $I \in \mathcal{M}_{n_{1}}(\mathbb{C})$ is the identity matrix.) It remains to be shown that the equality holds in (2.3) for then $X$ will be an operator module by [6].

So, let $a=\left[a_{1}, a_{2}\right] \in \mathcal{R}_{n_{1}+n_{2}}(A)$ and $b=\left[b_{1}, b_{2}\right]^{T} \in \mathcal{C}_{n_{1}+n_{2}}(B)$ be any contractions, where $a_{i} \in \mathcal{R}_{n_{i}}(A)$ and $b_{i} \in \mathcal{C}_{n_{i}}(B)(i=1,2)$. Suppose first that $A$ and $B$ are von Neumann algebras and let $a_{i}^{*}=u_{i}^{*}\left|a_{i}^{*}\right|$ and $b_{i}=v_{i}\left|b_{i}\right|$ be the usual polar decompositions, hence $\left|a_{i}^{*}\right|=\left(a_{i} a_{i}^{*}\right)^{1 / 2} \in A, u_{i} \in \mathcal{R}_{n_{i}}(A),\left|b_{i}\right|=\left(b_{i}^{*} b_{i}\right)^{1 / 2} \in B, v_{i} \in \mathcal{C}_{n_{i}}(B)$ and $\left\|u_{i}\right\|=1=\left\|v_{i}\right\|$. Then for arbitrary $x_{i} \in \mathcal{M}_{n_{i}}(X)(i=1,2)$ we have

$$
\begin{aligned}
\left\|a\left(x_{1} \oplus x_{2}\right) b\right\| & =\left\|a_{1} x_{1} b_{1}+a_{2} x_{2} b_{2}\right\|=\left\|\left|a_{1}^{*}\right|\left(u_{1} x_{1} v_{1}\right)\left|b_{1}\right|+\left|a_{2}^{*}\right|\left(u_{2} x_{2} v_{2}\right)\left|b_{2}\right|\right\| \\
& \leq\left\|\left|a_{1}^{*}\right|^{2}+\left|a_{2}^{*}\right|^{2}\right\|^{1 / 2} \max \left\{\left\|u_{1} x_{1} v_{1}\right\|,\left\|u_{2} x_{2} v_{2}\right\|\right\}\left\|\left|b_{1}\right|^{2}+\left|b_{2}\right|^{2}\right\|^{1 / 2} \\
& \leq\left\|a a^{*}\right\|^{1 / 2} \max \left\{\left\|x_{1}\right\|,\left\|x_{2}\right\|\right\}\left\|b^{*} b\right\|^{1 / 2} \leq \max \left\{\left\|x_{1}\right\|,\left\|x_{2}\right\|\right\}
\end{aligned}
$$

where for the first inequality we have used (2.1) and for the second inequality we have used the definition (2.2) of the norms on $\mathcal{M}_{n}(X)$. Since this holds for all contractions $a \in \mathcal{R}_{n_{1}+n_{2}}(A)$ and $b \in \mathcal{C}_{n_{1}+n_{2}}(B)$ it follows from the definition (2.2) of the norm in $\mathcal{M}_{n_{1}+n_{2}}(X)$ that $\left\|x_{1} \oplus x_{2}\right\| \leq \max \left\{\left\|x_{1}\right\|,\left\|x_{2}\right\|\right\}$. This proves that equality holds in (2.3) if $A$ and $B$ are von Neumann algebras. If $A$ and $B$ are general $C^{*}$-algebras, then note that for each $\varepsilon \in(0,1)$ we have $b_{i}=\left(v_{i}\left|b_{i}\right|^{\varepsilon}\right)\left|b_{i}\right|^{1-\varepsilon}$ and $v_{i}\left|b_{i}\right|^{\varepsilon} \in \mathcal{C}_{n_{i}}(B)$ since $\left|b_{i}\right|^{e}$ can be 
approximated by polynomials in $\left|b_{i}\right|$ with the zero constant term. Using a similar decomposition for $a_{i}^{*}$ and letting $\varepsilon \rightarrow 0$ at the end one can easily modify the above proof to the setting of general $C^{*}$-algebras.

Corollary 2.2. A left Banach module $X$ over a $C^{*}$-algebra $A$ is isometrically isomorphic to an operator A-module if and only if

$$
\left\|a_{1} x_{1}+a_{2} x_{2}\right\| \leq\left\|a_{1} a_{1}^{*}+a_{2} a_{2}^{*}\right\|^{1 / 2}\left(\left\|x_{1}\right\|^{2}+\left\|x_{2}\right\|^{2}\right)^{1 / 2}
$$

for all $a_{i} \in A$ and $x_{i} \in X(i=1,2)$. A similar result holds also for right modules.

Proof. If the condition (2.4) is satisfied for all $a_{i} \in A$ and $x_{i} \in X$ then for all $\lambda_{1}, \lambda_{2} \in \mathbb{C}$ we have

$$
\begin{aligned}
\left\|\lambda_{1} a_{1} x_{1}+\lambda_{2} a_{2} x_{2}\right\| & \leq\left\|a_{1} a_{1}^{*}+a_{2} a_{2}^{*}\right\|^{1 / 2}\left(\left|\lambda_{1}\right|^{2}\left\|x_{1}\right\|^{2}+\left|\lambda_{2}\right|^{2}\left\|x_{2}\right\|^{2}\right)^{1 / 2} \\
& \leq\left\|a_{1} a_{1}^{*}+a_{2} a_{2}^{*}\right\|^{1 / 2} \max \left\{\left\|x_{1}\right\|,\left\|x_{2}\right\|\right\}\left(\left|\lambda_{1}\right|^{2}+\left|\lambda_{2}\right|^{2}\right)^{1 / 2}
\end{aligned}
$$

Thus by Theorem $2.1 X$ is isometric to an operator $A$-module. This proves the corollary in one direction, the proof of the converse is trivial.

An operator $A, B$-bimodule $X$ on which the operator space structure satisfies (2.2) will be called a minimal operator $A, B$-bimodule (of a given Banach $A, B$-bimodule $X$ ).

For example, if $A$ and $B$ are $C^{*}$-subalgebras of $\mathrm{B}(\mathcal{H})$ with cyclic vectors, then $\mathrm{B}(\mathcal{H})$ (and consequently any subbimodule of $\mathrm{B}(\mathcal{H})$ ) is a minimal operator $A, B$-bimodule. To see this, let $x \in \mathcal{M}_{n}(\mathrm{~B}(\mathcal{H}))$, denote by $\xi_{0}$ and $\eta_{0}$ the cyclic vectors for $A$ and $B$ (respectively) and choose unit vectors $\xi=\left(a_{1} \xi_{0}, \ldots, a_{n} \xi_{0}\right)$ and $\eta=\left(b_{1} \eta_{0}, \ldots, b_{n} \eta_{0}\right)$ in $\mathcal{H}^{n}$ such that $\langle x \eta, \xi\rangle$ approximates $\|x\|$. Then, using an approximate variant of the polar decompositions $a=u|a|$ and $b=v|b|$, where $a=\left(a_{1}, \ldots, a_{n}\right)^{T} \in \mathcal{C}_{n}(A)$ and $b=\left(b_{1}, \ldots, b_{n}\right)^{T} \in \mathcal{C}_{n}(B)$ (as in [18, p. 48]), it follows easily that $\left\|u^{*} x v\right\|$ approximates $\|x\|$, where $u^{*}$ and $v$ are contractions in $\mathcal{R}_{n}(A)$ and $\mathcal{C}_{n}(B)$ (respectively).

As another example, each $C^{*}$-algebra $A$ is easily seen to be a minimal $A, A$-operator bimodule and so is any closed two-sided ideal $J$ in $A$ and also the quotient bimodule $A / J$.

Perhaps the simplest example of a Banach module over a $C^{*}$-algebra $A \neq \mathbb{C}$ which does not satisfy the condition (2.4) is the dual space $A^{\sharp}$ with the usual module structure (namely $(a \rho)(b)=\rho(b a), a, b \in A, \rho \in A^{\sharp}$ ). With $p \in A^{\sharp}$ a nontrivial projection and $\rho_{1}, \rho_{2} \in A^{\mathbb{\sharp}}$ normal states on $A^{\mathbb{t}}$ with the support $p$ and $p^{\perp}$ (respectively), we have $\left\|p \rho_{1}+p^{\perp} \rho_{2}\right\|=2$, but $\left\|p p^{*}+p^{\perp} p^{\perp *}\right\|^{1 / 2}\left(\left\|\rho_{1}\right\|^{2}+\left\|\rho_{2}\right\|^{2}\right)^{1 / 2}=\sqrt{2}$. Hence $A^{\sharp}$ does not satisfy the condition (2.4) as $A^{\sharp t}$-module, which easily implies that $A^{\sharp}$ can not satisfy (2.4) as $A$-module either. In the case $A=\mathcal{M}_{2}(\mathbb{C})$ the fact $A^{\mathbb{1}}$ with the standard dual operator space structure is not an operator $A$-module was observed by Blecher in [2].

Remark. If $X$ is a Banach $A, B$-bimodule satisfying the condition (2.1) (where $A$ 
and $B$ are $C^{*}$-algebras) then there is also a maximal operator $A, B$-bimodule structure on $X$, given by $\left\|\left[x_{i, j}\right]\right\|=\sup \left\|\left[\Phi\left(x_{i, j}\right)\right]\right\|\left(\left[x_{i, j}\right] \in \mathcal{M}_{n}(X)\right)$, where the supremum is over all contractive $A, B$-bimodule homomorphisms from $X$ into $\mathrm{B}(\mathcal{H})$ (where $\mathcal{H}$ is a Hilbert space on which $A$ and $B$ are represented, so that $\mathrm{B}(\mathcal{H})$ is an $A, B$-bimodule). This is completely analogous to the definition of a maximal operator space of a normed space (see [4] and [14]) and one can express these maximal norms for modules in a similar intrinsic way as in $[14,2.1]$, but we shall not study this maximal operator module structure here.

In the rest of this section we show by an example that for a non selfadjoint operator algebra $A$ the condition (2.4) is not always sufficient for a Banach $A$-module to have an isometric representation as an operator module. The appropriate module in the example is a finite dimensional Hilbert space. As a byproduct we shall find in the first two paragraphs of the example a very simple unital contractive representation of an operator algebra which is not completely contractive. (The first example of such a representation was found by Arveson in [1].) The example itself is not needed in the rest of the paper, so it may be skipped.

Example 2.3. Let $p \in \mathbb{R}, p \geq 1$ and let $n>p$ be an integer. Let $A \subseteq \mathcal{M}_{n+1}(\mathbb{C})$ be the (commutative) algebra of all matrices of the form

$$
\left[\begin{array}{cc}
\alpha & 0 \\
v & \alpha I
\end{array}\right]
$$

where $\alpha \in \mathbb{C}, I$ is the $n \times n$ identity matrix and $v \in \mathbb{C}^{n}$ is an arbitrary vector. Let $\varphi: A \rightarrow \mathcal{M}_{n+1}(\mathbb{C})$ be the homomorphism defined by

$$
\varphi\left(\left[\begin{array}{cc}
\alpha & 0 \\
v & \alpha I
\end{array}\right]\right)=\left[\begin{array}{cc}
\alpha & \frac{1}{\sqrt{p}} v^{T} \\
0 & \alpha I
\end{array}\right],
$$

where $v^{T}$ is the transpose of $v$. It is easy to verify that $\|\varphi\|=1$. Let $X=\mathbb{C}^{n+1}$ and define the $A$-module structure on $X$ by

$$
a \circ x=\varphi(a) x \quad(a \in A, x \in X) .
$$

Since $\varphi$ is a unital contractive homomorphism, $X$ is an essential Banach $A$-module.

Let $\left\{e_{i}: i=1, \ldots, n\right\}$ be the standard basis of $\mathbb{C}^{n}$ (regarded as the space of columns) and define $E_{i} \in A$ by

$$
E_{i}=\left[\begin{array}{ll}
0 & 0 \\
e_{i} & 0
\end{array}\right]
$$

Then 


$$
\left\|\left[E_{1}^{T}, \ldots, E_{n}^{T}\right]\right\|=\left\|\sum_{i=1}^{n} E_{i}^{T} E_{i}\right\|^{1 / 2}=\left(\sum_{i=1}^{n}\left\|e_{i}\right\|^{2}\right)^{1 / 2}=\sqrt{n}
$$

and

$$
\left\|\left[E_{1}, \ldots, E_{n}\right]\right\|=\left\|\sum_{i=1}^{n} E_{i} E_{i}^{T}\right\|^{1 / 2}=1,
$$

since $E_{i} E_{i}^{T}$ are mutually orthogonal projections. (Observe from this that the representation $\varphi$ is not completely contractive if $p=1$ and $n \geq 2$, in fact in this case $\varphi$ is not even 2-contractive.) If $\left(x_{1}, \ldots, x_{n}\right) \in\left(\mathbb{C}^{n+1}\right)^{n}$ is a unit vector at which the operator $\left[E_{1}^{T}, \ldots, E_{n}^{T}\right]$ attains its norm, we have

$$
\left\|\sum_{i=1}^{n} E_{i} \circ x_{i}\right\|=\left\|\sum_{i=1}^{n} \frac{1}{\sqrt{p}} E_{i}^{T} x_{i}\right\|=\sqrt{\frac{n}{p}}>1=\left\|\sum_{i=1}^{n} E_{i} E_{i}^{*}\right\|^{1 / 2}\left(\sum_{i=1}^{n}\left\|x_{i}\right\|^{2}\right)^{1 / 2},
$$

hence the module multiplication $A \times X \rightarrow X$ can not be completely contractive for any operator structure on $X$ such that the norm on $X$ coincides with the given one. Now we are going to show that nevertheless the condition (2.4) is satisfied if $p$ is chosen appropriately.

Given two elements

$$
a_{1}=\left[\begin{array}{cc}
\alpha_{1} & 0 \\
v_{1} & \alpha_{1} I
\end{array}\right] \text { and } a_{2}=\left[\begin{array}{cc}
\alpha_{2} & 0 \\
v_{2} & \alpha_{2} I
\end{array}\right]
$$

in $A$, the norm of the matrix $a_{1} a_{1}^{*}+a_{2} a_{2}^{*}$ can be computed explicitly. We assume that

$$
\beta:=\left|\alpha_{1}\right|^{2}+\left|\alpha_{2}\right|^{2} \neq 0
$$

If $\beta=0$ the computation is easier and is left to the reader. To facilitate the computation put

$$
\frac{1}{\beta}\left(\bar{\alpha}_{1} v_{1}+\bar{\alpha}_{2} v_{2}\right)=w_{1}, \quad \text { and } \quad \frac{1}{\beta}\left(-\alpha_{2} v_{1}+\alpha_{1} v_{2}\right)=w_{2}
$$

Then

$$
v_{1}=\alpha_{1} w_{1}-\bar{\alpha}_{2} w_{2}, \quad v_{2}=\alpha_{2} w_{1}+\bar{\alpha}_{1} w_{2}
$$

and

$$
a_{1} a_{1}^{*}+a_{2} a_{2}^{*}=\beta\left[\begin{array}{cc}
1 & w_{1}^{*} \\
w_{1} & w_{1} w_{1}^{*}+w_{2} w_{2}^{*}+I
\end{array}\right]
$$


To compute the norm of the matrix in (2.5), let $e_{1}$ and $e_{2}$ be two orthogonal unit vectors such that $w_{1}=\gamma e_{1}$ and $w_{2}=\mu e_{1}+v e_{2}$, where $\gamma, \mu$ and $v$ are scalars with $\gamma \geq 0$ and $v \geq 0$. Then the matrix in (2.5) is unitarily equivalent to the direct sum of the matrix

$$
\left[\begin{array}{ccc}
1 & \gamma & 0 \\
\gamma & \gamma^{2}+|\mu|^{2}+1 & \mu \nu \\
0 & \bar{\mu} \nu & v^{2}+1
\end{array}\right]
$$

and the $(n-2) \times(n-2)$ identity matrix. Put

$$
r=\left\|w_{1}\right\|^{2}=\gamma^{2} \quad \text { and } \quad s=\left\|w_{2}\right\|^{2}=|\mu|^{2}+v^{2} .
$$

The characteristic polynomial of the matrix (2.6) is (denoting $z=1-\lambda$ )

$$
F(z)=z^{3}+(r+s) z^{2}+\left(r s-r-\gamma^{2}|\mu|^{2}\right) z-\left(r s-\gamma^{2}|\mu|^{2}\right) .
$$

Let

$$
G(z)=z^{3}+(r+s) z^{2}+r(s-1) z-r s=(z+s)\left(z^{2}+r z-r\right)
$$

which is just the special case of $F(z)$ when $\gamma \mu=0$. The smallest zero of $G$ is $\min \left\{-s,-\frac{1}{2}\left(r+\sqrt{r^{2}+4 r}\right)\right\}$. Evidently $G(z) \leq F(z)$ if $z \leq 0$. Since for $z \rightarrow-\infty$ we have $F(z)<0$ and $G(z)<0$, the smallest zero of $F$ must be less than or equal to the smallest zero of $G$. Since the norm of the matrix in (2.5) is the same as the norm of the matrix (2.6), which is equal to its largest eigenvalue $(\lambda=1-z)$, we see that

$$
\left\|a_{1} a_{1}^{*}+a_{2} a_{2}^{*}\right\| \geq \beta\left[1+\max \left\{s, \frac{1}{2}\left(r+\sqrt{r^{2}+4 r}\right)\right\}\right] .
$$

To prove that the condition (2.4) is satisfied, let $x_{i} \in X(i=1,2)$ be such that $\left\|x_{1}\right\|^{2}+\left\|x_{2}\right\|^{2} \leq 1$. Then we have

$$
\begin{aligned}
\left\|a_{1} \circ x_{1}+a_{2} \circ x_{2}\right\| & \leq\left\|\varphi\left(a_{1}\right) \varphi\left(a_{1}\right)^{*}+\varphi\left(a_{2}\right) \varphi\left(a_{2}\right)^{*}\right\|^{1 / 2}\left(\left\|x_{1}\right\|^{2}+\left\|x_{2}\right\|^{2}\right)^{1 / 2} \\
& \leq\left\|\varphi\left(a_{1}\right) \varphi\left(a_{1}\right)^{*}+\varphi\left(a_{2}\right) \varphi\left(a_{2}\right)^{*}\right\|^{1 / 2}
\end{aligned}
$$

Using the previous notation, one easily computes that

$$
\varphi\left(a_{1}\right) \varphi\left(a_{1}\right)^{*}+\varphi\left(a_{2}\right) \varphi\left(a_{2}\right)^{*}=\beta\left[\begin{array}{cc}
1+\frac{1}{p}(r+s) & \frac{1}{\sqrt{p}} w_{1}^{T} \\
\frac{1}{\sqrt{p}} \bar{w}_{1} & I
\end{array}\right]
$$

and 


$$
\left\|\varphi\left(a_{1}\right) \varphi\left(a_{1}\right)^{*}+\varphi\left(a_{2}\right) \varphi\left(a_{2}\right)^{*}\right\|=\beta\left[1+\frac{1}{2 p}\left(r+s+\sqrt{\left.(r+s)^{2}+4 p r\right)}\right]\right.
$$

Now to prove that the condition (2.4) is satisfied, it suffices by (2.8) to show that $\left\|\varphi\left(a_{1}\right) \varphi\left(a_{1}\right)^{*}+\varphi\left(a_{2}\right) \varphi\left(a_{2}\right)^{*}\right\| \leq\left\|a_{1} a_{1}^{*}+a_{2} a_{2}^{*}\right\|$. For this, by (2.7) and (2.9) it suffices to show that for some $p>0$ (which determines the choice of the map $\varphi$ and the module $X$ ) the inequality

$$
\frac{1}{2 p}\left[r+s+\sqrt{(r+s)^{2}+4 p r}\right] \leq \max \{s, t\}
$$

holds for all $r, s \geq 0$, where $t=\frac{1}{2}\left(r+\sqrt{r^{2}+4 r}\right)$. It is possible to prove (by a tedious computation) that (2.10) is true if we choose $p=2$, but the proof is much simpler if we choose a larger $p$. So let $p=4$ and suppose that the inequality $(2.10)$ is not true. Then both $s$ and $t$ are smaller than the left side of $(2.10)$, hence so must be the convex combination $\frac{1}{4} s+\frac{3}{4} t$. Thus we have

$$
\frac{1}{4} s+\frac{3}{8}\left(r+\sqrt{r^{2}+4 r}\right)<\frac{1}{8}\left(r+s+\sqrt{(r+s)^{2}+16 r}\right) \leq \frac{1}{8}(2 r+2 s+4 \sqrt{r})
$$

which implies (after a simplification) that $r+3 \sqrt{r^{2}+4 r}<4 \sqrt{r}$, but this is clearly impossible for $r \geq 0$.

Problem. Let $A$ be a unital (non-selfadjoint) operator algebra and $X$ a left Banach $A$-module. Suppose that

$$
\left\|\sum_{i=1}^{n} a_{i} x_{i}\right\| \leq\left\|\sum_{i=1}^{n} a_{i} a_{i}^{*}\right\|^{1 / 2}\left(\sum_{i=1}^{n}\left\|x_{i}\right\|^{2}\right)^{1 / 2}
$$

for all finite sets $\left\{a_{1}, \ldots, a_{n}\right\} \subseteq A$ and $\left\{x_{1}, \ldots, x_{n}\right\} \subseteq X$. Is then $X$ necessarily isometrically isomorphic to an operator module?

\section{Normal modules}

Throughout this section $A$ and $B$ will be von Neumann algebras and $X$ an operator $A, B$-bimodule.

If $X$ as a Banach bimodule is a dual of some Banach $B, A$-bimodule $Y$ (in the sense that $X$ is the dual Banach space of $Y$ and $\langle a x b, y\rangle=\langle x, b y a\rangle$ for all $a \in A, b \in B$, $x \in X$ and $y \in Y)$, then we can introduce in $Y$ the so called $L_{1}$-matrically normed structure by $\|y\|=\sup \left\{|\langle x, y\rangle|: x \in \mathcal{M}_{n}(X),\|x\| \leq 1\right\}$ for all $y \in \mathcal{M}_{n}(Y)$, where $\langle x, y\rangle:=$ $\sum_{i, j=1}^{n}\left(x_{i, j}, y_{j, i}\right)$ with $x_{i, j}$ and $y_{i, j}$ the entries of $x$ and $y$, respectively (see [8]). The bimodule multiplication $B \times Y \times A \rightarrow Y$ is then easily seen to be completely contractive, hence so is the bimodule multiplication $A \times X \times B \rightarrow X$ if $X$ carries the 
new $L_{\infty}$-matrically normed structure defined by $\|x\|^{\prime}=\sup \left\{|\langle x, y\rangle|: y \in \mathcal{M}_{n}(Y),\|y\| \leq 1\right\}$ for $x \in \mathcal{M}_{n}(X)$. Obviously $\|x\|^{\prime} \leq\|x\|$ for all $x \in \mathcal{M}_{n}(X)$ and the equality holds here if $n=1$, hence by the minimality of $(X,\|\|)$, the equality must hold for all $n$. It follows that $X$ as an operator $A, B$-bimodule is the dual of $Y$ in the sense of [8] and we shall call such a bimodule $X$ a dual minimal operator $A, B$-bimodule. We do not assume in general that for all $x \in X$ the maps

$$
a \mapsto a x \quad \text { and } \quad b \mapsto x b
$$

from $A$ and $B$, respectively, to $X$ are weak* to weak* continuous. If the maps (3.1) are weak* to weak* continuous then by [8, Theorem 3.5] $X$ is a normal dual operator $A, B$-bimodule in the sense that there exist a Hilbert space $\mathcal{H}$, a complete weak* continuous isometry $\Phi: X \rightarrow \mathrm{B}(\mathcal{H})$ and faithful normal unital representations $\pi: A \rightarrow \mathrm{B}(\mathcal{H})$ and $\sigma: B \rightarrow \mathrm{B}(\mathcal{H})$ such that

$$
\Phi(a x b)=\pi(a) \Phi(x) \sigma(b) \quad \text { for all } a \in A, b \in B \text { and } x \in X
$$

A general (not necessarily dual) operator $A, B$-bimodule $X$ will be called normal if there exist a complete isometry $\Phi$ and representations $\pi$ and $\sigma$ as above, except that now we do not require that $\Phi$ is continuous in the weak* topology (there is no such topology on $X$ now).

If $X$ is a normal operator $A, B$-bimodule then clearly for each $x \in X$ the mappings

$$
a \mapsto\|a x\| \quad \text { and } \quad b \mapsto\|x b\|
$$

are lower semicontinuous in the weak* topology of $A$ and $B$, respectively.

Question. Is the lower semicontinuity of the maps (3.3) a sufficient condition for a minimal operator $A, B$-bimodule $X$ to be normal?

For general (not necessarily minimal) operator bimodules it is natural to require in this problem the lower semicontinuity of the mappings defined by (3.3) on $\mathcal{M}_{n}(A)$ and $\mathcal{M}_{n}(B)$ for all $x \in \mathcal{M}_{n}(X)$ and all $n$. We shall see below that the answer to the above question is affirmative in the case of one-sided modules (that is, if $A$ or $B$ is $\mathbb{C}$ ). In the case of bimodules we have to replace the semicontinuity of the maps (3.3) by an apparently stronger condition, to guarantee the normality of bimodules, but we do not have any concrete example of a minimal $A, B$-operator bimodule $X$ for which the maps (3.3) are lower semicontinuous and $X$ is not normal. Before proceeding with formal theorems, we shall now indicate how the results of [8] imply that the answer to the above question is affirmative if $X$ is a dual bimodule.

If $X$ is any dual operator space, then by [8, Theorem 3.3] $X$ can be represented isometrically and weak* continuously as a weak* closed subspace of $\mathrm{B}(\mathcal{H})$ for some Hilbert space $\mathcal{H}$; if in addition $X$ is a dual operator $A, B$-bimodule, then an argument in the proof of Theorem 3.4 in $[8$, p. 150] shows that there exist a Hilbert space $\mathcal{H}$, a 
weak* homemorphic complete isometry $\Phi: X \rightarrow \mathrm{B}(\mathcal{H})$ and two (not necessarily normal) unital representations $\pi: A \rightarrow \mathrm{B}(\mathcal{H})$ and $\sigma: B \rightarrow \mathrm{B}(\mathcal{H})$ such that (3.2) holds. We now replace $\pi$ and $\sigma$ with their restrictions to the invariant subspaces $\mathcal{H}_{1}=[\Phi(X) \mathcal{H}]$ and $\mathcal{H}_{2}=\left[\Phi(X)^{*} \mathcal{H}\right]$, respectively, and regard $\Phi(X)$ as a subspace in $\mathbf{B}\left(\mathcal{H}_{2}, \mathcal{H}_{1}\right)$. Then the left and the right annihilator of $\Phi(X)$ in $\mathbf{B}\left(\mathcal{H}_{1}\right)$ and $\mathbf{B}\left(\mathcal{H}_{2}\right)$ (respectively) is 0 . We claim that the two representations $\pi$ and $\sigma$ are necessarily normal if the mappings (3.3) are lower semicontinuous, hence $X$ is a normal dual operator $A, B$-bimodule. In fact, to prove that, say, $\pi$ is normal, it suffices to show that for each normal state $\omega$ on $\mathbf{B}\left(\mathcal{H}_{1}\right)$ the state $\omega \pi$ on $A$ is normal, which is equivalent to the condition that for each net of projections $\left\{e_{v}\right\}$ in $A$ increasing to the identity 1 the net $\left\{\omega \pi\left(e_{v}\right)\right\}$ converges to $\omega \pi(1)=1$ (see e.g. [10]). Thus, it suffices to show that the limit $f$ of the increasing net of projections $\left\{\pi\left(e_{v}\right)\right\}$ in $\mathrm{B}\left(\mathcal{H}_{1}\right)$ is equal to 1 . For each $x \in X$ we have that $f^{\perp} \Phi(x) \in \Phi(X)$ (since $\Phi(X)$ is weak* closed in $\mathrm{B}\left(\mathcal{H}_{2}, \mathcal{H}_{1}\right)$ ), hence $f^{\perp} \Phi(x)=\Phi\left(x_{0}\right)$ for some $x_{0} \in X$. Since $\left\|e_{v} x_{0}\right\|=\left\|\Phi\left(e_{v} x_{0}\right)\right\|=\left\|\pi\left(e_{v}\right) \Phi\left(x_{0}\right)\right\|=\left\|\pi\left(e_{v}\right) f^{\perp} \Phi(x)\right\|=0$ for all $v$, it follows from the assumed semicontinuity of the norm that $x_{0}=0$. Thus $f^{\perp} \Phi(x)=0$ for all $x \in X$, which implies $f^{\perp}=0$ since the left annihilator of $\Phi(X)$ in $\mathrm{B}\left(\mathcal{H}_{1}\right)$ is 0 . This proves that $f=1$, as required. So $\pi$ and $\sigma$ are now normal representations and consequently $X$ is a normal operator $A, B$-bimodule.

The set of all linear functionals on the Haagerup tensor product $A \otimes^{h} B$ which (as bilinear forms on $A \times B$ ) are normal in each variable separately will be denoted by $\mathrm{Bil}^{\sigma}(A, B)$. (We refer to [18] and [4] for the definition of the Haagerup tensor product. An explicit characterisation of functionals in $\operatorname{Bil}^{\circ}(A, B)$ can be found in [7], but we shall nod need this characterisation here.) The topology on $A \otimes^{h} B$ determined by the family of seminorms $w \mapsto|\vartheta(w)|\left(\vartheta \in \mathrm{Bil}^{\sigma}(A, B)\right)$ will be called the normal weak topology.

Given a Banach $A, B$-bimodule $Y$, let $\tilde{Y}$ be the Banach $B, A$-subbimodule of the dual bimodule $Y^{\sharp}$ consisting of all functionals $\omega$ on $Y$ such that for each $y \in Y$ the two maps $a \mapsto \omega(a y)$ and $b \mapsto \omega(y b)$ on $A$ and $B$ (respectively) are normal. We shall need the following simple lemma (a variant of which for one-sided modules has been proved already in [12]).

Lemma 3.1. If $Z$ is a subbimodule of a Banach $A, B$-bimodule $Y$ then each $\omega \in \tilde{Z}$ can be extended to some $\theta \in \tilde{Y}$ such that $\|\theta\|=\|\omega\|$.

Proof. Let $\phi$ be any linear extension of $\omega$ to $Y$ such that $\|\phi\|=\|\omega\|$ and for each $x \in X$ put $\theta(x)=\left(\phi_{x}\right)_{\text {nor }}(1,1)$, where $\phi_{x}: A \times B \rightarrow \mathbb{C}$ is the bilinear map defined by $\phi_{x}(a, b)=\phi(a x b)$ and $\left(\phi_{x}\right)_{\text {nor }}$ is the part of $\phi_{x}$ which is normal in each variable separately. This normal part is defined in the following way: first we extend $\phi_{x}$ to a bilinear form $\psi$ on $A^{\sharp \sharp} \times B^{\sharp t}$ which is normal in each variable separately, where $A^{\sharp t}$ and $B^{ \pm t}$ are the universal von Neumann algebras of the von Neumann algebras $A$ and $B$ (such an extension is possible and unique by [18, Lemma 3.3.2]); then we define $\left(\phi_{x}\right)_{\text {nor }}$ by $\left(\phi_{x}\right)_{\text {nor }}(a, b)=\psi(p a, q b)$, where $p$ and $q$ are the central projections in $A^{\text {th }}$ and $B^{\mathrm{tg}}$ (respectively) such that $A \cong p A^{t:}$ and $B \cong q B^{\sharp 5}$ (see [10, Section 10.1] for more details about this projections if necessary). It is now easy to verify that the so defined map $\theta$ has the required properties. 
We shall denote $\overline{\bar{Y}}$ by $\hat{Y}$. If $X$ is an operator $A, B$-bimodule, then $\hat{X}$ is an operator $A, B$-bimodule and the natural map $\imath: X \rightarrow \hat{X}$ is a completely contractive homomorphism of bimodules. Then $X$ is a normal operator $A, B$-bimodule if and only if $t$ is a complete isometry. This is proved in [12] for one-sided modules, but the proof for bimodules is the same. If $X$ carries the minimal operator $A, B$-bimodule structure and $l$ is an isometry, then of course $\imath$ must be a complete isometry.

Recall that each element in the Haagerup tensor product $A \otimes^{h} B$ can be written in the form $\sum_{i=1}^{\infty} a_{i} \otimes b_{i}=: a \odot b$, where $a$ ( $b$, respectively) is an infinite row (column) with the entries in $A(B)$ such that the series $\sum_{i=1}^{\infty} a_{i} a_{i}^{*}\left(\sum_{i=1}^{\infty} b_{i}^{*} b_{i}\right)$ is norm convergent.

Proposition 3.2. Let $A$ and $B$ be von Neumann algebras and $X$ an operator $A, B$ bimodule. Then $X$ is a normal operator $A, B$-bimodule if and only if for each $n \in \mathbb{N}$ and each $x \in \mathcal{M}_{n}(X)$ the function

$$
p_{x}: \mathcal{M}_{n}(A) \otimes^{h} \mathcal{M}_{n}(B) \rightarrow \mathbb{R}, \quad p_{x}(a \odot b)=\|a x b\|
$$

is lower semicontinuous in the normal weak topology of $\mathcal{M}_{n}(A) \otimes^{h} \mathcal{M}_{n}(B)$. If $X$ is a minimal operator $A, B$-bimodule then it suffices to require the above condition for $n=1$ only.

Proof. For simplicity of notation we shall prove the theorem for minimal bimodules, the proof for general bimodules is essentially the same. If $X$ is normal, we may assume that $X, A$ and $B$ are contained in $\mathrm{B}(\mathcal{H})$ for some Hilbert space $\mathcal{H}$ and the algebraic operations in $A, B$ and $X$ coincide with the usual operations in $\mathrm{B}(\mathcal{H})$. Then for each normal functional $\omega$ on $\mathrm{B}(\mathcal{H})$ and each $x \in X$ the functional

$$
\vartheta: A \otimes^{h} B \rightarrow \mathbb{R}, \quad \vartheta(a \otimes b)=\omega(a x b)
$$

is clearly normal in each variable $a$ and $b$ separately, hence $\vartheta \in \operatorname{Bil}^{\circ}(A, B)$. If $\left\{a_{v} \odot b_{v}\right\}$ is a net in $A \otimes^{h} B$ converging to an element $a \odot b$ in the normal weak topology, then in particular $\lim \vartheta\left(a_{v} \odot b_{v}\right)=\vartheta(a \odot b)$. This implies (since $\omega$ can be any normal linear functional on $\mathrm{B}(\mathcal{H})$ ) that the net $\left\{a_{v} x b_{v}\right\}$ converges to $a x b$ in the weak* topology of $\mathrm{B}(\mathcal{H})$, hence $\|a x b\| \leq \liminf _{v}\left\|a_{v} x b_{y}\right\|$, which proves the required semicontinuity of the function $p_{x}$.

Assume now that all the functions $p_{x}$ are lower semicontinuous in the normal weak topology of $A \otimes^{h} B$. To prove that $X$ is a normal $A, B$-bimodule, it suffices by the remarks preceding the proposition to show that the natural map $\imath: X \rightarrow \hat{X}$ is an isometry. By hypothesis for each $x \in X$ the set

$$
S=\left\{w \in A \otimes^{h} B: p_{x}(w) \leq 1\right\}
$$

is closed in the normal weak topology and clearly $S$ is also convex and balanced. Assume that $x \neq 0$ and let $\varepsilon>0$. Since $p_{x}(1 \otimes 1)=\|x\|$, the element $\frac{1+e}{|x|} 1 \otimes 1$ is not in $S$, hence by the geometric variant of the Hahn-Banach theorem there exists 
$\vartheta \in \operatorname{Bil}^{\sigma}(A, B)$ such that $|\vartheta(w)| \leq 1$ for each $w \in S$ and $\vartheta\left(\frac{1+\varepsilon}{n \times \|} 1 \otimes 1\right)>1$. This implies that $|\vartheta(w)| \leq p_{x}(w)$ for each $w \in A \otimes^{h} B$ and $\vartheta(1 \otimes 1)>\frac{\|x\|}{1+\varepsilon^{*}}$. Thus, we can define a functional $\psi$ on the subbimodule $A x B$ of $X$ by

$$
\psi(a \times b)=\Im(a \odot b)
$$

and we have $|\psi(a x b)| \leq\|a x b\|$ for all $a \odot b \in A \otimes^{h} B$ (hence $\|\psi\| \leq 1$ ) and $\psi(x)>\frac{\|x\|}{1+\varepsilon}$. Since $\vartheta \in \operatorname{Bil}^{\sigma}(A, B)$, the functional $\psi$ is in $(A x B)^{-}$. By Lemma $3.1 \psi$ can be extended to a functional in $\tilde{X}$ without increasing the norm. Since $\varepsilon>0$ in this argument was arbitrary, we see that the natural contraction $\imath: X \rightarrow \hat{X}$ must indeed be isometric.

Theorem 3.3. A left operator module $X$ over a von Neumann algebra $A$ is normal if and only if for each $n \in \mathbb{N}, x \in \mathcal{M}_{n}(X)$ and each net $\left\{e_{v}\right\}$ of projections in $\mathcal{M}_{n}(A)$ converging to the identity 1 the equality

$$
\|x\|=\lim _{v}\left\|e_{v} x\right\|
$$

holds. Moreover, if $A$ is $\sigma$-finite, it suffices to check this condition for increasing sequences of projections in $A$ instead of general nets. If $X$ is a minimal left operator $A$ module, then it suffices to require the condition for $n=1$ only.

Proof. Again, for simplicity of notation we shall consider only minimal modules. We need only to prove the sufficiency of the above condition for $X$ to be normal. By Proposition 3.2 (applied to the $A, \mathbb{C}$-bimodule $X$ ) it suffices to show that for each $x \in X$ the function

$$
p_{x}: A \rightarrow \mathbb{R}, \quad p_{x}(a)=\|a x\|
$$

is lower semicontinuous in the weak* topology of $A$, which is equivalent to the requirement that the convex set $S=\{a \in A:\|a x\| \leq 1\}$ is weak* closed in $A$. By the Krein-Smulian theorem [15] it suffices to prove that the intersection of $S$ with each closed ball $A_{r}$ in $A$ with the centre 0 and radius $r$ is weak* closed or, equivalently, closed in the *-strong operator topology of $A_{r}$. So, let $\left\{a_{v}\right\}$ be a net in $S \cap A_{r}$ converging to an element $a \in A_{r}$ in the $*$-strong operator topology. Using the noncommutative Egoroff theorem (see [19]), it follows that each strong neighbourhood $U$ of the identity 1 in $A$ contains a projection $e_{U} \in A$ such there is a sequence $\left\{a_{U, k}\right\} \subseteq\left\{a_{v}\right\}$ for which the sequence $\left\{e_{U} a_{U, k}: k=1,2, \ldots\right\}$ converges to $e_{U} a$ in the norm topology. This implies that $\left\|e_{U} a x\right\| \leq 1$ since $\left\|a_{U, k} x\right\| \leq 1$ for all $k$. Since the projections $e_{U}$ ( $U$ a neighbourhood of 1 in the strong operator topology) form a net converging to 1 , it follows now from the hypothesis of the Theorem that $\|a x\| \leq 1$, which concludes the proof that $S$ is weak* closed in $A$.

If $A$ is $\sigma$-finite, we may choose a sequence of basic neighbourhoods $U_{n}$ of 1 in the strong topology of the ball $A_{r}$ and then for each $n$ a projection $e_{n}=e_{U_{n}} \in U_{n}$ as above. In addition, we may require that $e_{n+1} \geq e_{n}$ for each $n$. Indeed, assuming inductively that 
$e_{1}, \ldots, e_{n}$ and a sequence $\left\{a_{k}\right\} \subseteq\left\{a_{v}\right\}$ have been already constructed such that the sequence $\left\{e_{n}\left(a_{k}-a\right)\right\}$ is norm convergent to 0 as $k \rightarrow \infty$, we apply the Egoroff theorem to the sequence $\left\{e_{n}^{\perp}\left(a_{k}-a\right)\left(a_{k}-a\right)^{*} e_{n}^{\perp}\right\}_{k \in N}$ in the von Neumann algebra $e_{n}^{\perp} A e_{n}^{\perp}$ to find an appropriate subprojection $f \leq e_{n}^{\perp}$ and a subsequence of $\left\{a_{k}\right\}$, and then we put $e_{n+1}=e_{n}+f$. Then it follows as in the previous paragraph that the set $S$ is weak* closed.

\section{Quotient modules}

Throughout this section $A$ will be a von Neumann algebra.

If $X$ is an operator $A$-module we can define the so called $A$-topology on $X$ by the family of seminorms

$$
s_{\omega}(x)=\inf \left\{\omega\left(a a^{*}\right)^{1 / 2}\|y\|: x=a y, a \in \mathcal{R}_{n}(A), y \in \mathcal{C}_{n}(X), n \in \mathbb{N}\right\},
$$

where $\omega$ is any normal positive linear functional on $A$. It is proved in [12] that (4.1) is indeed a seminorm and that in (4.1) it suffices to take $n=1$ and $y \in X, a \in A$ with $0 \leq a \leq 1$, without changing the value of $s_{\omega}$. This implies that the $A$-topology is independent of the norms on $\mathcal{M}_{n}(X)$ for $n>1$. The fact that $X$ is an operator module is required only to prove that the quantities (4.1) are indeed seminorms and to establish some useful properties of this topology.

The continuous linear functionals in the $A$-topology of $X$ are just the norm bounded functionals $\theta$ on $X$ such that for each $x \in X$ the functional $a \mapsto \theta(a x)$ is normal on $A$ (see [12]). As in the previous section, we denote the set of all such functionals by $\tilde{X}$. This characterisation of continuous functionals implies that for each normal dual operator $A$-module $X$ and each submodule $Y$ of $X$ the closure of $\mathcal{M}_{n}(Y)$ in the $\mathcal{M}_{n}(A)$ topology of $\mathcal{M}_{n}(X)$ is equal to $\mathcal{M}_{n}\left(\bar{Y}^{A}\right)$, where $\bar{Y}^{A}$ is the closure of $Y$ in the $A$-topology of $X$.

The question, which we would like to discuss in this section is the following.

Question. Let $X$ be a normal dual operator $A$-module and $Y$ a norm closed submodule of $X$. Under what conditions is the quotient operator module $X / Y$ normal?

If $X / Y$ is normal then for each $x \in X$ the map

$$
\alpha_{x}: a \mapsto\|a x+Y\|
$$

must be lower semicontinuous in the weak* topology of $A$ by Section 3. (Of course the same semicontinuity condition must hold also for the analogous maps defined on $\mathcal{M}_{n}(A)$ for all $x \in \mathcal{M}_{n}(X)$ and $\left.n=1,2, \ldots\right)$ In particular, for each $x \in X$ the left ideal

$$
\{a \in A: a x \in Y\}=\alpha_{x}^{-1}(0)
$$


in $A$ must be weak* closed. By $[12,2.1,5.3]$ the condition that this ideals are weak* closed for all $x \in X$ is equivalent to the requirement that $Y$ is closed in the $A$-topology of $X$ (such submodules $Y$ are called strong in [11]). Thus, a necessary condition for $X / Y$ to be a normal operator $A$-module is that $Y$ is closed in $X$ in the $A$-topology. In certain special circumstances this condition is also sufficient for $X / Y$ to be normal.

Proposition 4.1. Let $A$ be a $\sigma$-finite von Neumann algebra contained in a concrete $C^{*}$-algebra $X \subseteq \mathrm{B}(\mathcal{H})$ and let $J$ be a norm closed two-sided ideal of $X$. Suppose that $X$ contains the closure $Y$ of $J$ in the A-topology. (This is satisfied, for example, if $X$ is a von Neumann algebra since the A-topology is stronger than the weak* topology.) Then $X / Y$ is a normal operator left A-module.

Proof. By Theorem 3.3 it suffices to show that for each $m=1,2 \ldots$, each $x \in \mathcal{M}_{m}(X)$ and each increasing sequence of projections $f_{n} \in \mathcal{M}_{m}(A)$ converging to 1 the equality

$$
\alpha:=\lim \left\|f_{n} \dot{x}\right\|=\|\dot{x}\|
$$

holds, where $\dot{x} \in \mathcal{M}_{m}(X / Y) \cong \mathcal{M}_{m}(X) / \mathcal{M}_{m}(Y)$ is the coset of $x$. As we have already noted in the beginning of this section, $\mathcal{M}_{m}(Y)$ is just the closure of $\mathcal{M}_{m}(J)$ in the $\mathcal{M}_{m}(A)$-topology; moreover, since $\mathcal{M}_{m}(J)$ is a two-sided ideal in $\mathcal{M}_{m}(X)$, we may restrict our attention (for simplicity of notation) to the case $m=1$, the proof for general $m$ is the same. For each $n$ let $e_{n}=f_{n}-f_{n-1}$ (where $f_{0}:=0$ ), so that $\left\{e_{n}: n=1,2, \ldots\right\}$ is an orthogonal sequence of projections in $A$ with the sum 1 and $f_{n}=\sum_{j=1}^{n} e_{j}$. Choose any $\beta>\alpha$. Since $\left\|f_{n} \dot{x}\right\|<\beta$, for each $n$ there exists an $y_{n}=f_{n} y_{n} \in Y$ such that $\left\|f_{n} x-y_{n}\right\|<\beta$ and the sequence $y_{n}$ is bounded.

We claim that for each $n$ there exists an orthogonal sequence $\left\{e_{n, j}: j=1,2, \ldots\right\}$ of projections in $A$ such that

$$
\sum_{j=1}^{\infty} e_{n, j}=e_{n} \quad \text { and } \quad e_{n, j} y_{k} \in J \text { for all } n, j, k=1,2, \ldots
$$

To see this, we first note that the closure in the $A$-topology of the two-sided ideal $J$ in $X$ is necessarily a norm closed right ideal in $X$. (This can easily be proved directly and follows also from [12, 2.2 and 5.3].) Hence the element $z:=\sum_{k=1}^{\infty} 2^{-k} y_{k} y_{k}^{*}$ is in $Y$. Consequently $e_{n} z \in Y$ for each $n$ and by [12, 2.2 and 5.3] there exists an orthogonal sequence $\left\{e_{n, j}: j=1,2, \ldots\right\}$ of projections in $A$ with sum $e_{n}$ such that $e_{n, j} z \in J$ for each $j$. Then $e_{n, j} z e_{n, j} \in J$, hence $e_{n, j} y_{k} y_{k}^{*} e_{n, j} \in J$ for all $k$, hence $e_{n, j} y_{k} \in J$ for all $n, j, k$ since $J$ is a two-sided ideal in $X$. (We have used the well known fact that $w^{*} w \in J$ implies $w \in J$ for $w \in X$, where $J$ may be any norm closed two-sided ideal in a $C^{*}$-algebra $X$.) This proves the claim.

For each $n$ let $p_{n}=\sum_{i+j \leq n} e_{i, j}$, so that $\left\{p_{n}: n=1,2, \ldots\right\}$ is an increasing sequence of projections in $A$ converging to 1 . Then we have $p_{n} y_{n} \in J$ and $\left\|p_{n}\left(x-y_{n}\right)\right\| \leq\left\|f_{n}\left(x-y_{n}\right)\right\|<\beta$ for all $n$, hence 


$$
\left\|p_{n} x+J\right\|<\beta
$$

Put $q_{n}=p_{n}-p_{n-1}$ (with $p_{0}=0$ ) and $x_{n}=q_{n} x$. We shall construct inductively a sequence of elements $z_{n}=q_{n} z_{n} \in J$ such that

$$
\left\|\sum_{i=1}^{n}\left(x_{i}-z_{i}\right)\right\| \leq \beta
$$

for all $n$. Since the series $\sum x_{i}$ converges in the strong operator topology and $x_{i}=q_{i} x_{i}$ and $z_{i}=q_{i} z_{i}$, where the $q_{i}$ 's are mutually orthogonal projections, (4.3) will imply that the series $\sum z_{i}$ converges strongly to an element $z$ (namely, the partial sums of the series $\sum\left(x_{i}-z_{i}\right)$ are bounded in norm by $\beta$ and the terms $x_{i}-z_{i}$ have mutually orthogonal ranges). Since $q_{i} z=z_{i} \in Y$ and $Y$ is closed in the $A$-topology, it follows from [12] that $z \in Y$. (Alternatively, it is not hard to show directly that the partial sums of the series $\sum z_{i}$ converge to $z$ in the $A$-topology.) Since $z \in Y$ and (4.3) implies that $\|x-z\| \leq \beta$, it follows that $\|\dot{x}\| \leq \beta$. But this holds for all $\beta>\alpha$, hence $\|\dot{x}\| \leq \alpha\left(=\lim \left\|f_{n} \dot{x}\right\|\right)$, which will prove the required lower semicontinuity. So it remains to construct the required sequence $\left\{z_{i}: i=1,2, \ldots\right\}$.

Suppose by induction that we have already constructed the elements $z_{i}=q_{i} z_{i} \in J$ for $i=1, \ldots, n-1$ such that

$$
\left\|\sum_{i=1}^{n-1}\left(x_{i}-z_{i}\right)\right\| \leq \beta
$$

Put $b=\sum_{i=1}^{n-1}\left(x_{i}-z_{i}\right)^{*}\left(x_{i}-z_{i}\right)$, so that $0 \leq b \leq \beta^{2}$. Denote by $\tilde{v}$ the coset in $X / J$ of an element $v \in X$. From (4.2) it follows that

$$
\sum_{i=1}^{n} \tilde{x}_{i}^{*} \tilde{x}_{i} \leq \beta^{2},
$$

hence $\tilde{x}_{n}^{*} \tilde{x}_{n} \leq \beta^{2}-\tilde{b}$. Therefore we can lift $\tilde{x}_{n}$ to an element $v_{n} \in X$ such that $v_{n}^{*} v_{n} \leq \beta^{2}-b$ (see $\left[10\right.$, p. 290]). Finally, let $z_{n}=x_{n}-v_{n}$. Then $z_{n} \in J$ and $\left(x_{n}-z_{n}\right)^{*}\left(x_{n}-z_{n}\right) \leq \beta^{2}-b$. By the definition of $b$ this implies that $\left\|\sum_{i=1}^{n}\left(x_{i}-z_{i}\right)\right\| \leq \beta$ (since the elements $x_{i}-z_{i}$ have mutually orthogonal ranges), which concludes the proof.

Since in the special case $X \subseteq A$ (where the $A$-topology and the weak* topology have the same closed convex sets) and in the case $A=\mathbb{C}$ and $X$ arbitrary (where the $A$ topology is just the norm topology) the condition that $Y$ is closed in the $A$-topology is also sufficient for $X / Y$ to be normal, we might expect naively (encouraged by Proposition 4.1) that this may be so in general. But the following example shows that there exist normal operator modules $Y \subseteq \mathrm{B}(\mathcal{K})\left(\mathcal{K}\right.$ a Hilbert space) over $\ell_{\infty}$ such that the $\ell_{\infty}$-module $\mathrm{B}(\mathcal{K}) / Y$ is not normal although (surprisingly) the module $\bar{Y} / Y$ is normal, where $\bar{Y}$ is the closure of $Y$ in the weak ${ }^{*}$ topology of $B(\mathcal{K})$. 
Example 4.2. Let $\mathcal{H}$ be any infinite dimensional Hilbert space and $\mathcal{H}^{\infty}=\mathcal{H} \otimes \ell_{2}$. Let $\ell_{\infty}$ act on $\ell_{2}$ in the usual way and let $A=1_{\mathcal{H}} \otimes \ell_{\infty} \subseteq \mathrm{B}\left(\mathcal{H}^{\infty}\right)$. Then $X:=\mathrm{B}\left(\mathcal{H}^{\infty}\right)$ is a normal operator left $A$-module. Let $Y=\mathrm{K}(\mathcal{H})^{\infty} \subseteq \mathrm{B}\left(\mathcal{H}^{\infty}\right)$ be the usual $\ell_{\infty}$-direct sum of countably many copies of the ideal $\mathrm{K}(\mathcal{H})$ of all compact operators on $\mathcal{H}$. Clearly $Y$ is a left $A$-submodule of $X$ and we claim that $Y$ is closed in the $A$-topology of $X$. To see this, let $\left\{e_{i}: i \in \mathbb{N}\right\}$ be the set of minimal projections in $A$ with the sum 1 . Observe that for each $x \in X$ the condition that $e_{i} x \in Y$ for all $i \in \mathbb{N}$ implies that $x \in Y$; by [12, 2.1 and 5.3] (or directly) this implies that $Y$ is closed as required.

Let $\left\{E_{n}: n=1,2, \ldots\right\}$ be a set of orthogonal projections in $\mathrm{K}(\mathcal{H})$ with the sum 1 . Let $x \in X\left(=\mathcal{M}_{\infty}(\mathcal{H})\right)$ be defined by the operator matrix

$$
x=\left[\begin{array}{cccc}
1_{\mathcal{H}} & 0 & 0 & \ldots \\
E_{1} & 0 & 0 & \ldots \\
E_{2} & 0 & 0 & \ldots \\
\vdots & \vdots & \vdots &
\end{array}\right]
$$

We claim that the function $a \mapsto\|a \dot{x}\|$ is not lower semicontinuous in the weak* topology of $A$, where $\dot{x}$ is the coset of $x$ in $X / Y$, hence $X / Y$ is not a normal operator left $A$-module. To see this, let $f_{n}=e_{1}+\ldots+e_{n} \in A$; thus $f_{n}$ is the diagonal operator matrix in $M_{\infty}(\mathrm{B}(\mathcal{H})$ ) which has 1 on the first $n$ diagonal positions and 0 on the remaining positions. Clearly the sequence $\left\{f_{n}\right\}$ converges to 1 strongly. Since for each $c \in \mathrm{K}(\mathcal{H})$ the operator $\tilde{c}=c \oplus 0 \oplus 0 \oplus \ldots$ is in $Y$, we have $\left\|f_{n} \dot{x}\right\| \leq\left\|f_{n}(x-\tilde{c})\right\|$ for each $c \in \mathrm{K}(\mathcal{H})$. By choosing for $c$ the projection $c=E_{1}+\ldots+E_{n}$, it follows that

$$
\left\|f_{n} \dot{x}\right\| \leq\left\|(1-c)^{2}+c\right\|^{1 / 2}=1
$$

for all $n=1,2, \ldots$ On the other hand we shall now compute that $\|\dot{x}\| \geq \sqrt{2}$, which will prove that the map $a \mapsto\|a \dot{x}\|$ is not lower semicontinuous in the weak ${ }^{*}$ topology of $A$ since $f_{n} \nearrow 1$. Indeed, for each $y \in Y$ the norm of the operator matrix $x-y$ is greater than or equal to the norm of its first column, hence

$$
\|x-y\| \geq\left\|(1-d)^{*}(1-d)+\sum_{i=1}^{\infty} E_{i}^{*} E_{i}\right\|^{1 / 2}=\left\|(1-d)^{*}(1-d)+1\right\|^{1 / 2},
$$

where $d \in \mathbf{K}(\mathcal{H})$ is the first component of $y$. Since the norm of an operator dominates the norm of the corresponding coset in the Calkin algebra, it follows from the last estimate that $\|\dot{x}\| \geq \sqrt{2}$ (in fact $\|\dot{x}\|=\sqrt{2}$ since $\|x\|=\sqrt{2}$ ).

Although the operator $A$-module $X / Y$ is not normal, it follows from Proposition 4.1 that $\mathbf{B}(\mathcal{H})^{\infty} / Y$ is normal (at least if $\mathcal{H}$ is separable), and here $\mathbf{B}(\mathcal{H})^{\infty}$ is just the weak* closure of $Y$.

Acknowledgements. The author is grateful to $M$. Hladnik for reading the preliminary version of this paper. 


\section{REFERENCES}

1. W. B. ARveson, Subalgebras of $C$-algebras, Acta Math. 123 (1969), 141-224.

2. D. P. Blecher, The standard dual of an operator space, Pacific J. Math. 153 (1992), $15-30$.

3. D. P. BLECHER, A completely bounded characterisation of operator algebras, Math. Ann. 303 (1995), 227-239.

4. D. P. Blecher and V. I. Paulsen, Tensor products of operator spaces, J. Funct. Anal. 99 (1991), 262-292.

5. D. P. Blecher, Z.-J. RuAN and A. M. Sinclatr, A characterisation of operator algebras, J. Funct. Anal. 89 (1990), 188-201.

6. E. Christensen, E. G. Effros and A. Sinclair, Completely bounded multilinear maps and $C^{*}$-algebraic cohomology, Invent. Math. 90 (1987), 279-296.

7. E. G. Efrros and A. Kishimoto, Module maps and Hochschild-Johnson cohomology, Indiana Univ. Math. J. 36 (1987), 257-276.

8. E. G. EFFros and Z.-J, RUAN, Representation of operator bimodules and their applications, J. Operator Theory 19 (1988), 137-157.

9. E. G. Efrros and Z.-J. RuAN, On the abstract characterisation of operator spaces, Proc. Amer. Math. Soc. 119 (1993), 579-584.

10. R. V. KADISON and J. R. RINGROSE, Fundamentals of the theory of operator algebras, Vols. 1, 2 (Academic Press, London, 1983, 1986).

11. B. Magajna, Strong operator modules and the Hagerup tensor product, Proc. London Math. Soc. 74 (1997), 201-240.

12. B. Magajna, A topology for operator modules over $W^{*}$-algebras, J. Funct. Anal. 154 (1998), 17-41.

13. V. I. Paulsen, Completely bounded maps and dilations (Research Notes in Math., Vol. 146, Pitman, London, 1986).

14. V. I. PAULSEN, The maximal operator space of a normed space, Proc. Edinburgh Math. Soc. 39 (1996), 309-323.

15. G. K. Pedersen, Analysis now (Graduate Texts in Math. 118, Springer-Verlag, Berlin, 1989).

16. G. PISIER, Similarity problems and completely bounded maps (Lecture Notes in Math. 1618, Springer-Verlag, Berlin, 1996).

17. Z. J. Ruan, Subspaces of $C^{*}$-algebras, J. Funct. Anal. 76 (1988), 217-230.

18. A. M. Sinclair and R. R. SMith, Hochschild cohomology of von Neumann algebras (London Math. Soc. Lecture Note Series, Vol. 203, Cambridge Univ. Press, Cambridge, 1995).

19. M. TAKESAKI, Theory of operator algebras I (Springer-Verlag, New York, 1979). 
20. N. T. Varoupoulos, A theorem on operator algebras, Math. Scand. 37 (1975), 173-182.

Note. After this paper had been submitted for publication we received an unpublished manuscript from B. E. Johnson in which he also obtained a characterisation of Banach bimodules over a $C$-algebra that can carry the structure of operator bimodules, but his method is completely different from the proof of Theorem 2.1 .

Department of Mathematics

UNIVERSITY OF LJUBLJANA

JADRANSKA 19

LJUBLJANA 1000

SLOVENIA

E-mail address: Bojan.Magajna@fmf.uni-lj.si 